\section{ANNUAL Further
REVIESS}

Click here for quick links to Annual Reviews content online, including:

- Other articles in this volume

- Top cited articles

- Top downloaded articles

- Our comprehensive search

\title{
Emerging Molecular Targets for the Treatment of Nonalcoholic Fatty Liver Disease
}

\author{
Giovanni Musso, ${ }^{1}$ Roberto Gambino, ${ }^{2}$ \\ and Maurizio Cassader ${ }^{2}$ \\ ${ }^{1}$ Gradenigo Hospital, Turin, Italy; email: giovanni_musso@yahoo.it \\ ${ }^{2}$ Department of Internal Medicine, University of Turin, Italy
}

Annu. Rev. Med. 2010. 61:375-92

The Annual Review of Medicine is online at med.annualreviews.org

This article's doi:

10.1146/annurev.med.60.101107.134820

Copyright (c) 2010 by Annual Reviews. All rights reserved

0066-4219/10/0218-0375\$20.00

\section{Key Words}

NASH, farnesoid $\mathrm{X}$ receptor (FXR), pregnane $\mathrm{X}$ receptor (PXR), incretin, microRNA, AMP-activated kinase

\begin{abstract}
Nonalcoholic fatty liver disease (NAFLD) is characterized by hepatic fat accumulation in the absence of significant ethanol consumption, viral infection, or other specific causes of liver disease. Currently the most common chronic liver disease, affecting $30 \%$ of the Western world, NAFLD may progress to cirrhosis and end-stage liver disease and may increase the risk of developing diabetes and cardiovascular disease. Although its pathogenesis is unclear, NAFLD is tightly associated with insulin resistance and the metabolic syndrome. No established treatment exists, and current research is targeting new molecular mechanisms that underlie NAFLD and associated cardiometabolic disorders. This review discusses some of these emerging molecular mechanisms and their therapeutic implications for the treatment of NAFLD: microRNAs, incretin analogs/antagonists, liver-specific thyromimetics, AMP-activated protein kinase activators, and nuclear receptors farnesoid $\mathrm{X}$ receptor and pregane $\mathrm{X}$ receptor.
\end{abstract}




\section{INTRODUCTION}

NAFLD:

nonalcoholic fatty liver disease

NASH: nonalcoholic steatohepatitis
Nonalcoholic fatty liver disease (NAFLD) is characterized by hepatic fat accumulation in the absence of significant ethanol consumption, viral infection, or other specific causes of liver disease. Currently the most common chronic liver disease in the world, NAFLD affects $30 \%$ of the Western adult population (1) and encompasses a spectrum of histological severity ranging from simple steatosis to nonalcoholic steatohepatitis (NASH), the latter progressing to cirrhosis in $10 \%-25 \%$ of cases over $8-10$ years (2). Although its pathogenesis is unclear, NAFLD is tightly associated with overall and central adiposity, insulin resistance, metabolic syndrome, and type 2 diabetes mellitus.

\section{MICRoRNA BIOGENESIS AND REGULATORY FUNCTION}

MiRNAs are initially transcribed by RNA polymerase II or III in the nucleus to form large pri-miRNA transcripts, which may be several hundred or thousands of nucleotides long, and are capped (MGpppG) and polyadenylated. The pri-miRNAs are processed in the nucleus by the RNase III enzyme Drosha and by the dsRNA (double-stranded RNA)-binding protein Pasha (also known as DGCR8), into smaller pre-miRNAs, which fold into stem-loop hairpin structures. RanGTP and exportin 5 transport the pre-miRNA into the cytoplasm. Subsequently, another RNase III enzyme, Dicer, processes the pre-miRNA to generate a transient nucleotide duplex. The duplex is loaded into the miRNA-associated miRISC (multiprotein RNA-induced silencing complex), which includes the Argonaute proteins (AGO). One strand of the miRNA is preferentially retained in this complex and becomes the mature miRNA; the opposite strand, known as the passenger strand or miRNA*, is eliminated from the complex. Some intronic miRNA precursors can bypass Drosha processing to produce miRNAs by Dicer, possibly an alternative pathway for miRNA biogenesis. The mature miRNA binds to complementary sequences in the 30 untranslated regions (30UTRs) of the target mRNAs to regulate gene expression in one of two ways: miRNAs that bind to mRNA targets with imperfect complementarity block target gene expression via translational silencing, whereas miRNAs binding their mRNA targets with perfect complementarity enhance target gene expression.
Currently there is no established treatment for NAFLD. Weight loss through lifestyle changes is difficult to achieve and maintain, and thiazolidinediones (a class of insulin sensitizers), a promising drugs for the treatment of NAFLD/NASH, pose weight-gain and longterm safety issues. Therefore, basic research is exploring new molecular mechanisms to target for the treatment of NAFLD and associated disorders.

\section{MicroRNAS AND NAFLD}

MicroRNAs (miRNAs) are a large class of genomically encoded 22-nucleotide-long RNAs that regulate target mRNAs in plants and animals through an antisense mechanism. Since the identification of the first miRNA, lin-4, in the nematode Caenorbabditis elegans (3), investigators have identified 695 miRNAs in humans, 488 in mouse, and 152 in the fly Drosophila melanogaster (4). It is increasingly understood that miRNA maturation is complex and highly modulated, subjecting these small posttranscriptional regulators of gene expression themselves to extensive posttranscriptional control. Each miRNA has several target genes, possibly dozens or hundreds, involved in diverse developmental and cellular processes, including cellular metabolism proliferation, differentiation, and apoptosis (5). Binding of the target gene results in modulation of the target mRNA through mechanisms that have not been fully elucidated but seem to involve translational repression, deadenylation and degradation of the target mRNAs (see sidebar MicroRNA Biogenesis and Regulatory Function). MicroRNA dysregulation has been implicated in various diseases, particularly cancers $(6,7)$. Accordingly, much effort has been put into developing profiling methods for miRNA expression patterns as experimental tools for the biologist and potential diagnostic and therapeutic tools for the clinician.

In liver diseases, most research on miRNAs focuses on their role in hepatocarcinogenesis. Microarray analysis and quantitative real-time polymerase chain reaction (RT-PCR) disclosed 
Table 1 Dysregulated miRNAs in liver diseases

\begin{tabular}{|c|c|c|}
\hline Disease & Dysregulated miRNA & Target gene/cellular function \\
\hline Hepatocarcinoma & $\begin{array}{l}(+): 10 \mathrm{~b}, 18,20,21 \\
221 / 222,224 \\
(-): 122,145,150,199 \mathrm{a} \\
199 \mathrm{~b}, 200 \mathrm{~b}, 214,223\end{array}$ & $\begin{array}{l}\text { 21: promotes cell invasion, migration, and growth via repression of the tumor } \\
\text { suppressor PTEN } \\
\text { 122: suppresses cyclin G1 expression; binds HCV-RNA enhancing its replication }\end{array}$ \\
\hline Colangiocarcinoma & $(+): 21,23 a, 27,141,200 b$ & 21: promotes gemcitabine resistance \\
\hline & & $\begin{array}{l}\text { 21: suppresses PTEN expression } \\
\text { 107/103: modulates LDLRP-B (deleted in tumors), transforming growth factor- } \beta \\
\text { receptor III, fibroblast growth factor } 2 \\
\text { 122: modulates cholesterol and fatty acid synthesis } \\
\text { 135a: modulates apoptosis inhibitor } 5 \\
\text { 200b: modulates LDLRP-B, insulin receptor substrate } 1 \text {, tumor necrosis factor } \\
\text { receptor } 11 \mathrm{~b} \text { (osteoprotegerin) }\end{array}$ \\
\hline
\end{tabular}

Abbreviations/symbols: (-), downregulated; (+), upregulated; LDLRP-B, low-density lipoprotein receptor-related protein-B; PGC-1 $\alpha$, peroxisome proliferator-activated receptor- $\gamma$ coactivator- $1 \alpha$; PTEN, phosphatase and tensin homolog.

aberrant miRNA expression profiles in primary hepatocarcinoma (HCC) compared with normal liver tissue $(8,9)$ (Table 1). MiRNA-21 promotes hepatocyte invasion, migration, and growth via repression of the tumor suppressor phosphatase and tensin homolog (PTEN), whose targeted deletion in murine hepatocytes leads to the development of HCCs (10), and the miRNAs 221-222 are associated with the outcome of HCCs. Among miRNAs downregulated in HCCs, miRNA-122 has received much attention because it is expressed specifically in the liver and accounts for $70 \%$ of the total liver miRNAs. Among the validated targets of miRNA-122, cyclin G1 and hepatitis $\mathrm{C}$ virus (HCV) have significance in HCCs. Indeed, cyclin G1 levels are increased in HCCs, and experimental models of hepatocarcinogenesis demonstrated that loss of cyclin G1 is associated with a significantly lower tumor incidence after carcinogen treatment, whereas HCV represents a predisposition factor for HCC. MiRNA122 binds to the $5^{\prime}$ noncoding region of the $\mathrm{HCV}$ RNA genome, enhancing viral RNA replication (11). Consistently, miRNA-122 is downregulated in most of the mixed-etiology $\mathrm{HCCs}$ but upregulated in HCV-related HCCs, and interferon- $\beta$ reduces hepatic miRNA-122 levels $(12,13)$.

The role of miRNAs in NAFLD is currently being investigated, as well.

In human primary hepatocytes and in rats, the addition of monounsaturated olive oil upregulated the expression of miRNA-21, which bound to PTEN mRNA and induced its degradation. This in turn led to PTEN downregulation and hepatocyte fat infiltration (14). This study provides evidence for dietary regulation of miRNA-21 expression and may further link dietary fat excess to different liver disorders, given the emerging role of the tumor suppressor PTEN in the pathogenesis of NAFLD, NASH, and HCC (15).

Microarray and RT-PCR technology were utilized to detect dysregulated miRNAs in a rat model of NAFLD (16). Compared to healthy animals, there were 58 miRNAs upregulated and 51 miRNAs downregulated in NAFLD, most of which were differentially expressed in simple steatosis versus steatohepatitis. Putative target genes of dysregulated miRNAs were involved in hepatocyte proliferation, apoptosis, inflammation, and glucose and lipid metabolism (Table 1). 
FAS: fatty acid synthase

GLP-1: glucagon-like peptide-1

GIP: glucosedependent insulinotropic polypeptide
Finally, the hepatic expression profile of 474 miRNAs was assessed in patients with NASH. Compared with healthy controls, 23 miRNAs were differentially expressed in the liver of NASH subjects (17). Opposite to mouse models, hepatocyte miRNA-122 expression was $63 \%$ lower in human NASH; reduced miRNA122 expression was associated with an increased expression of several target miRNA-122 lipogenic genes, including fatty acid synthase (FAS), 3-hydroxy-3-methyl-glutarylcoenzyme A reductase (HMG-CoA reductase), and their transcriptional activators, the sterol-responseelement binding proteins $1 \mathrm{c}$ and 2 (SREBP1c, SREBP-2). Intriguingly, in a subsequent experiment with cultured human hepatocytes, in vitro silencing and overexpression of miR-122 by reverse transfection were able to functionally modulate the expression of all these target genes and proteins involved in lipid synthesis.

The clinical implications of these data are twofold. First, the miRNA profile may serve as a diagnostic and prognostic biomarker in NAFLD, helping to noninvasively differentiate simple steatosis, which has a benign course, from progressive NASH, which can progress to cirrhosis and end-stage liver disease. Second, recent findings that antisense oligonucleotides can selectively block specific miRNAs in vitro and in vivo triggered efforts to explore miRNAs as a potential new class of therapeutics. A recent study demonstrated that intravenous injection of a short locked nucleic-acid-modified oligonucleotide ("LNA-antimir") could block miR-122 activity in monkey, accompanied by a dose-dependent and long-lasting lowering of plasma cholesterol, without evidence of toxicity or carcinogenity (18). A phase I safety study in humans is ongoing. In high-fatfed mice, silencing miR-122 by a $20-\mathrm{OMe}$ phosphorothioate-modified antisense oligonucleotide specific to miR-122 ("antagomir") resulted in a significant reduction of hepatic steatosis, which was associated with reduced cholesterol synthesis rates and stimulation of hepatic fatty acid oxidation (19, 20). Conversely, restoring miRNA function by the exogenous delivery of a synthetic miRNA mimic might have therapeutic benefit where tumor-suppressor miRNAs are underrepresented; a recent study demonstrated that viral delivery of let-7 inhibited tumor formation in mouse models of lung cancer (21).

These encouraging data suggest a miRNAmodulating strategy may prove an effective therapeutic approach for many metabolic and malignant diseases, including NASH. The upcoming challenges are (a) to identify all targets of deregulated miRNAs in these diseases and assess which miRNAs or antimicroRNAs can be used; $(b)$ to ensure both the stability of the modulators in the body and, most importantly, delivery to, and uptake by, the organ or cells of interest; and $(c)$ last, but not least, to assess carefully long-term safety of this approach.

\section{INCRETIN}

\section{ANALOGS/ANTAGONISTS}

Incretin mimetics have received much attention in recent years because of their ability to stimulate pancreatic $\beta$-cell insulin secretion and growth, and they have been recently approved for the treatment of diabetes. Different strategies for enhancing incretin action have been proposed. These agents include glucagon-like peptide-1 (GLP-1) analogs, glucose-dependent insulinotropic polypeptide (GIP) agonists, and dipeptidyl peptidase-4 (DPP-4) inhibitors, which inhibit the enzyme responsible for incretin inactivation.

It is increasingly recognized GLP-1 and GIP exert relevant extrahepatic actions that go well beyond their antidiabetic action (reviewed in 22) and may differentially affect their overall metabolic effects (Table 2).

The rationale for employing GLP-1 analogs in NAFLD lies in their weight-losing and intrahepatic effects (Table 2). Furthermore, reduced incretin action has been demonstrated in nondiabetic nonobese patients with NASH (23), thus prompting evaluation of GLP-1 analogs in fatty liver. In ob/ob mice, two months of exendin-4 induced a dose-dependent weight loss (7\%-14\% compared with placebo) and a significant improvement in hepatic steatosis, 
Table 2 Similarities and differences between incretins GLP-1 and GIP

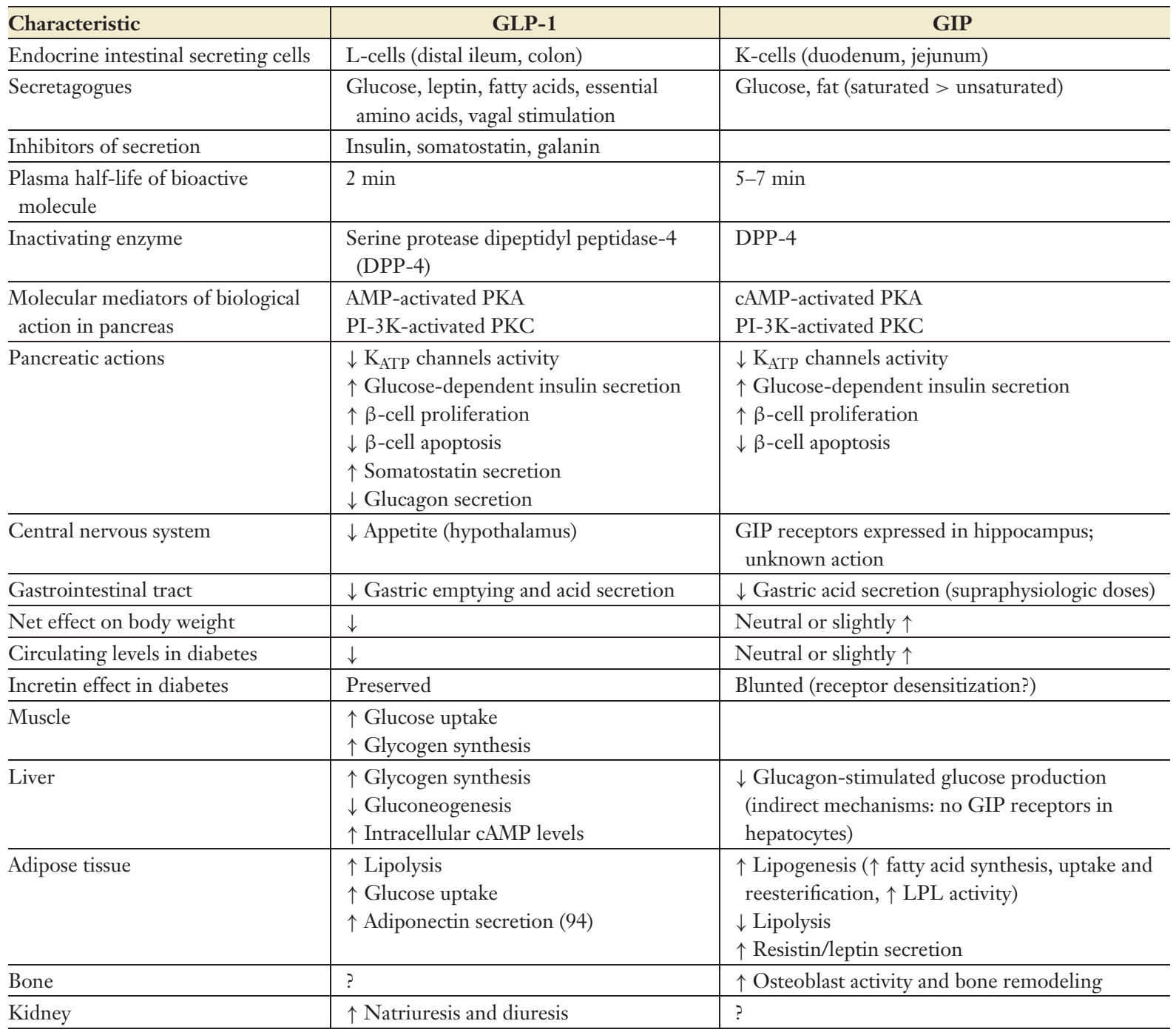

Abbreviations: cAMP, cyclic adenosine-monophosphate; PKA, protein kinase A; PKC, protein kinase C; LPL, lipoprotein lipase; PI-3K, phosphoinositide 3-kinase.

insulin sensitivity, and plasma adipokine profile. These changes were associated with a marked increase in hepatocyte cAMP production and fatty acid oxidative pathways and with a decrease in hepatic lipogenesis and oxidative stress (24). In humans, Tushuizen et al. reported a 3.6-fold reduction in liver fat following 44 weeks of exenatide therapy in a diabetic man (25). In an open-label extension of three randomized placebo-controlled trials enrolling
217 overweight diabetic subjects, exenatide for three years was safe and well-tolerated, and it achieved a mean $5.3-\mathrm{kg}$ weight loss as well as a significant reduction of serum transaminases, normalizing ALT levels in $41 \%$ of patients (26).

Although these data suggest a possible role for GLP-1 agonists in the treatment of NAFLD, preclinical evidence suggests GIP may promote obesity-associated metabolic disorders (27). GIP operates as a key modulator 
FFA: free fatty acid of lipid metabolism by stimulating glucose uptake, lipoprotein lipase activity, free fatty acid (FFA) synthesis, and incorporation in adipocytes (28). Furthermore, GIP administration stimulated resistin and leptin secretion in cultured adipocytes, an effect totally prevented by knocking out GIP receptor $(29,30)$. Consistent with these findings, acute and chronic administration of GIP, but not of GLP-1, induced fatty liver and other obesity-associated metabolic disorders in mice, whereas GIP antagonism reversed liver, muscle, and adipose tissue fat infiltration and ameliorated associated metabolic disturbances induced by high-fat diet in mice $(31,32)$.

Dietary fat is the most potent stimulator of GIP secretion. Saturated fat exerts higher stimulatory effects than unsaturated fat, and excessive saturated fat intake predisposes to diabetes, cardiovascular disease, and liver fat infiltration (33-37). Together these data suggest GIP may mediate the deleterious metabolic effects of a high-fat diet and may modulate adipocyte adipokine secretion, independently of its incretin effect on $\beta$-cell function (38).

Consistent with preclinical data, plasma GIP response to fat ingestion is exaggerated in nondiabetic nonobese patients with NASH and is associated with reduced $\beta$-cell function and incretin effect, as well as an unfavorable lipoprotein and adipokine profile (39). These findings suggest GIP may be an important mediator of the adipocyte response to nutritional excess, through molecular mechanisms still largely undefined, and may in part account for the increased metabolic risk of NAFLD.

The differential metabolic actions of GIP agonists, GLP-1 analogs, and DPP-4 inhibitors have been assessed in high-fat-fed mice, where the three classes of agents exerted different effects on $\beta$-cell function, insulin sensitivity, body weight, and adipokine levels. GLP1 analog exendin- 4 reduced food intake, and both exendin-4 and DPP-4 inhibitor prevented weight gain and ameliorated glucose tolerance and insulin sensitivity; GIP agonism did not prevent weight gain and was associated with worsened insulin sensitivity, increased circulating leptin and resistin, and a less pronounced improvement in glucose tolerance (40).

Therapeutically, as incretin analogs are evaluated in NAFLD, the relative merits of inhibition versus activation of GIP signaling should be considered. Specifically, while circulating levels of GLP-1 are reduced and pancreatic sensitivity to its incretin actions is preserved in obesity and diabetes, GIP has lost most of its insulin-stimulatory action, and its noxious extrapancreatic effects may offset the benefit of a blunted incretin effect. Although GLP-1agonists may be potentially effective and harmless, the benefits of GIP antagonism may overcome ablation of the insulin-releasing GIP component of the entero-insular axis, which is already failing in obesity and diabetes; therefore a strategy with mixed GLP-1 agonism and GIP antagonism may be more effective for the treatment of NAFLD and obesity-related disorders (27).

\section{LIVER-SPECIFIC THYROMIMETICS}

The antiobesity and low-density lipoprotein (LDL)-cholesterol-lowering effects of thyroid hormones have been known for years, but their pharmacological use has been limited by their untoward effects on bone and heart and skeletal muscle. Recent research has developed drugs that lack these adverse effects and harness the beneficial effects of thyroid hormone. The development of these drugs, termed thyromimetics, was made possible by the existence of distinct thyroid hormone receptor (TR) subtypes with different tissue-specific distribution (41). There are two subtypes of TRs, TR $\alpha$ and $\operatorname{TR} \beta$, predominantly localized in the heart and liver, respectively. Splice variants of each gene generate different TR isoforms, four of which (TR $\alpha 1, T R \alpha 2$, TR $\beta 1$, and $T R \beta 2)$ are expressed at the protein level in vivo (42).

Selective TR $\beta 1$ agonists have been synthesized and evaluated in preclinical trials and in a short clinical trial, showing their lipid-lowering properties and safety (43). The liver specificity 
of these drugs is achieved through the selective hepatic distribution of phosphonic acid: A phosphonate-containing TR $\beta 1$ agonist is taken up predominantly by the liver with a high firstpass extraction and converted into the active form in hepatic microsomes by cytochrome P450 3A.

Recently, the main compound of this type, MB07811, has been evaluated in different animal models of hepatic steatosis, including ob/ob mice, Zucker diabetic rats, and mice with diet-induced obesity (44). At the end of treatment, liver triglyceride content was reduced by $>66 \%$ in both normal and obese animals. A marked reduction was also observed in plasma cholesterol and triglyceride levels. Hepatic selectivity of MB07811 was demonstrated by showing that this agent did not affect circulating FFA levels, epidydimal fat pad and heart mass, or pituitary thyroid-stimulating hormone mRNA. Intrahepatic mechanisms of action of MB07811 included an increased rate of mitochondrial $\beta$-oxidation, as assessed by (a) an increase in the mRNA levels of carnitine palmitoyltransferase-1 (CPT-1), a rate-limiting enzyme in the fatty acid oxidation cycle; (b) elevated levels of short and intermediate-length acyl-carnitine species in plasma; $(c)$ increased mitochondrial respiration rates observed in ex vivo liver preparations; and $(d)$ increased activity of hepatic mitochondrial glycerol3-phosphate dehydrogenase (mGPDH), an enzyme involved in energy production and dissipation. A decrease in hepatic lipogenesis, as suggested by a reduction in SREBP-1c mRNA, and in hepatic lipase activity, as suggested by reduced levels of apolipoprotein C3 (apoC3, an inhibitor of lipoprotein lipase), might also contribute to the antisteatotic effects of this agent. These findings were independently reproduced by Perra et al. in choline-methionine-deficient (CMD) rat, an animal model of nutritional steatohepatitis (45). Importantly, in this study GC-1, another TR $\beta 1$-selective activator, ameliorated not only steatosis but also markers of hepatic lipoperoxidation and liver injury, suggesting these agents have the potential to reverse not only fat infiltration but also the progressive features of steatohepatitis. How TR mimetics can ameliorate hepatocyte oxidative stress and necroinflammation warrants further investigation. It has been proposed that T3 agonists generate "futile" cycles, including the glucose-6-phosphate cycle, the pyruvate/malate and pyruvate/citrate shuttles, the pentose-phosphate cycle, and the fructose6-phosphate/1,6-diphosphate cycles. These futile cycles reduce oxidative stress in two ways: They increase ATP cycling, which enhances oxidative phosphorylation and decreases the formation of reactive oxygen species (ROS); and they supply the NADPH necessary for the regeneration of reduced glutathione (GSH) and other reducing substances, thus facilitating the scavenging of ROS (46).

Collectively, the results of these studies show promise for the treatment of NAFLD and associated metabolic disorders, but efficacy and safety of this class of agents in humans need further evaluation.

\section{AMP-ACTIVATED PROTEIN KINASE}

The discovery that many of the beneficial effects of metformin, physical exercise, caloric restriction, leptin, and adiponectin-mediated actions of thiazolidinediones converge on the enzyme AMP-activated protein kinase (AMPK) has renewed interest in the modulation of the activity of this kinase (see sidebar Structure and Molecular Activators of AMPK). Pharmacological AMPK activators are being developed for the treatment of multiple metabolic disorders, including NAFLD (47).

Regulation of AMPK activity involves both direct allosteric activation by AMP and reversible phosphorylation of AMPK $\alpha$ subunit on Thr172 by upstream kinases. The combination of the allosteric and phosphorylation effects amplifies kinase activity by $>1000$-fold, allowing a fine integrated sensing of cellular metabolism in response to small changes in energy status. Under conditions of high energy demand, intracellular ATP is reduced, AMP rises, and the AMP/ATP ratio provides a very
AMPK: adenyl monophosphateactivated protein kinase 


\section{STRUCTURE AND MOLECULAR ACTIVATORS OF AMPK}

AMPK is a heterotrimeric serine/threonine kinase composed of a catalytic $\alpha$ subunit and regulatory $\beta$ and $\gamma$ subunits. Multiple genes encode the three subunits $(\alpha 1, \alpha 2, \beta 1, \beta 2, \gamma 1, \gamma 2, \gamma 3)$, allowing 12 heterotrimeric combinations, with splice variants. Of the 12 theoretically possible complexes only 3 were detected in human skeletal muscle $(\alpha 2 \beta 2 \gamma 1 \gg \alpha 2 \beta 2 \gamma 3=\alpha 1 \beta 2 \gamma 1)$. Serine/threonine kinase activity of AMPK is possessed by $\alpha$ subunits, which contain a threonine residue (Thr172) whose phosphorylation is required for AMPK activation. The $\beta$ subunits contain a $\mathrm{C}$-terminal region required for the association with $\alpha$ and $\gamma$ subunits and a central region that allows AMPK complex to bind glycogen, which explains the tight regulation of glycogen metabolism by AMPK. The $\gamma$ subunits contain four tandem repeats, known as the cystathionine $\beta$-synthase (CBS) motif, which bind two molecules of AMP or ATP in a mutually exclusive manner. Binding of AMP (on the $\gamma$ subunit) activates AMPK allosterically and promotes phosphorylation of the $\alpha$ subunit (on Thr172) by upstream kinases as protein kinase LKB1 or the $\mathrm{CaMKK} \beta$ (calmodulin-dependent protein kinase kinase). AMP binding also inhibits Thr172 dephosphorylation by protein phosphatase, while binding of ATP strongly inhibits the activation of AMPK. sensitive indication of cellular energy balance (48).

In the latest years, there has been increased interest in developing drugs that mimic the benefits of calorie restriction and physical exercise, which are the mainstay treatment for metabolic disorders but often cannot be achieved or maintained. Preclinical studies assessed the effects of pharmacological activation of AMPK on different organs and functions; the compound AICAR (5-aminoimidazole-4carboxamide-1- $\beta$-D-ribonucleoside), metabolized to ZMP, which is an analog of AMP, has been most extensively tested. In normal and insulin-resistant obese rats, infusion of AICAR suppressed hepatic glucose production, mostly through the phosphorylation and inactivation of a transcriptional coactivator, transducer of regulated CREB activity 2 (TORC2) (49, 50). TORC2 mediates
CREB-dependent transcription of peroxisomeproliferator-activated receptor- $\gamma$ coactivator $1 \alpha(\mathrm{PGC}-1 \alpha)$ and its gluconeogenic enzymes phosphoenolpyruvate carboxykinase (PEPCK) and glucose-6-phosphatase (G6Pase).

Reduced hepatic glucose output was coupled with enhanced glucose disposal by skeletal muscle, which was mediated by increased translocation of glucose transporter GLUT4 to cell membranes, enhanced mitochondrial biogenesis, and enhanced glucose oxidation. These effects occur through a different mechanism than the insulin-signaling pathway, involving the phosphorylation of TBC1D1, a Rab-GTPase-activating protein that regulates the translocation of GLUT4 from intracellular vesicles to the plasma membrane by maintaining G-proteins named Rab in a GDP-bound state. The phosphorylation and subsequent inactivation of TBC1D1 allow the release and translocation of GLUT4 from the vesicle (51).

Besides glucose homeostasis, AMPK also regulates hepatic lipid metabolism. Its activation by AICAR, alpha-lipoic acid, or oltipraz has been shown to decrease liver fat content in lean and obese rodents (52-54). These effects were mediated by a direct phosphorylation and inactivation of 3-hydroxy-3-methylglutarylcoenzyme A reductase (HMG-CoA reductase) and acetyl-CoA carboxylase (ACC), key enzymes in hepatic cholesterol and fatty acid synthesis, and by inhibition of SREBP1c-mediated transcription of key lipogenic enzymes, including fatty acid synthase, ACC, stearoyl-CoA desaturase-1, and ATP-binding cassette transporter A1 (55). Inhibition of ACC by AMPK leads to a fall in malonyl-CoA levels and subsequently removes the inhibition to mitochondrial fatty acid $\beta$-oxidation, eventually reducing excessive storage of triglycerides. Furthermore, AMPK activation directly leads to a significant activation of PGC- $1 \alpha$-mediated mitochondrial biogenesis, increasing mitochondrial number and enzymes in hepatocytes (56).

Other effects of AMPK activators include central appetite regulation, myocardial preconditioning and protection against ischemia and 
Table 3 Activators, molecular mediators, and effects of AMP kinase in different tissues and organs

\begin{tabular}{|c|c|c|c|}
\hline Activators & Tissue & Molecular target & Effect \\
\hline \multirow{4}{*}{$\begin{array}{l}\text { Physical exercise } \\
\text { Starvation } \\
\text { Biguanides, TZD } \\
\text { (adiponectin-mediated) } \\
\text { Adiponectin } \\
\text { Leptin } \\
\text { Ghrelin } \\
\text { AICAR } \\
\text { Thienopyridones } \\
\text { Dithiolethiones (oltipraz) } \\
\text { Alpha-lipoic acid } \\
\text { Polyphenols }(95,96)\end{array}$} & Skeletal muscle & $\begin{array}{l}\uparrow \text { GLUT- } 4 \text { translocation } \\
\uparrow \text { Hexokinase II } \\
\uparrow \text { PGC- } 1 \alpha \text {-mediated } \\
\text { mitochondrial biogenesis (97) }\end{array}$ & $\begin{array}{l}\uparrow \text { Glucose uptake and oxidation } \\
\uparrow \text { Insulin sensitivity }\end{array}$ \\
\hline & Liver & $\begin{array}{l}\uparrow \text { Phosphorylation of HMG-CoA } \\
\text { reductase/PK/ACC } \\
\downarrow \text { SREBP-1c-mediated lipogenesis } \\
\uparrow \mathrm{PGC}-1 \alpha \text {-mediated } \\
\text { mitochondrial biogenesis }\end{array}$ & $\begin{array}{l}\downarrow \text { Glucose production } \\
\downarrow \text { Lipogenesis and cholesterol synthesis } \\
\uparrow \text { Mitochondrial biogenesis and fatty acid } \\
\beta \text {-oxidation }\end{array}$ \\
\hline & Heart & $\begin{array}{l}\uparrow \text { Glucose utilization } \\
\downarrow \text { Myocyte hypertrophy }\end{array}$ & $\begin{array}{l}\text { Protects myocytes against ischemia } \\
\text { (ischemic preconditioning) } \\
\downarrow \text { Cardiac hypertrophy }\end{array}$ \\
\hline & Hypothalamus & $\begin{array}{l}\uparrow \text { Neuropeptide Y } \\
\uparrow \text { Agouti-related peptide (AgRP) } \\
\uparrow \text { Mitochondrial fatty acid } \\
\beta \text {-oxidation }\end{array}$ & $\uparrow$ Appetite \\
\hline
\end{tabular}

\begin{abstract}
Abbreviations: AICAR, 5 -amino-imidazole-4-carboxamide ribonucleoside; SREBP-1c, sterol regulatory element binding protein-1c; PGC-1 $\alpha$, peroxisome proliferator-activated receptor- $\gamma$ coactivator-1 $\alpha$; HMG-CoA reductase, 3 -hydroxy-3-methylglutaryl coenzyme A reductase; PK, pyruvate kinase; ACC, acetyl-CoA carboxylase.
\end{abstract}

hypertrophy (57-59), and endothelial function improvement through nitric-oxide-synthasedependent and -independent mechanisms (60). The benefits of AMPK activators on $\beta$-cell function are less well established (61, Table 3).

It is worth noting that the effects of AMPK activators are insulin independent and thus preserved in insulin-resistant-diabetic and obese subjects, such as those with NAFLD. These encouraging preclinical data need confirmation in human trials. The effects of AMPK activators on hepatic cellular mechanisms, other than lipogenesis, involved in necroinflammation and fibrogenesis are currently unknown, and such effects are crucial for the treatment of progressive NASH. In this context, AICAR infusion significantly reduced hepatic glucose output and whole-body lipolysis, decreasing blood glucose and FFA levels in a small group of diabetic subjects (62).

\section{NUCLEAR TRANSCRIPTION FACTORS: FARNESOID $\mathrm{X}$ RECEPTOR}

Originally described as a farnesol-activated receptor, farnesoid X receptor (FXR) has been long known for its function of bile acid sensor in enterohepatic tissues (63). FXR has emerged in recent years as a master regulator of lipid and glucose homeostasis and of inflammatory processes at hepatic and extrahepatic sites, and a number of synthetic FXR agonists are being tested for the treatment of different hepatic and metabolic disorders $(64,65)$.

Two FXR genes have been identified, $F X R \alpha$ and $F X R \beta$. FXR $\alpha$ encodes four isoforms $(\mathrm{FXR} \alpha 1, \mathrm{FXR} \alpha 2, \mathrm{FXR} \alpha 3$, and FXR $\alpha 4)$ as a result of the use of alternative-RNA splicing.

FXR $\alpha$ is expressed mainly in the liver, intestine, kidney, and adrenal glands, and at much lower levels in adipose tissues. The second FXR 
gene, $F X R \beta$, is a lanosterol sensor that encodes a functional protein in rodents but not in humans.

FXR is an obligate partner of the 9cis-retinoic acid receptor RXR (retinoid X receptor). The FXR/RXR heterodimer modulates gene expression through binding to specific DNA sequences called FXR response elements. Upon ligand binding, FXR undergoes conformational changes to release corepressors such as nuclear corepressor and recruit coactivators such as PGC- $1 \alpha$, CARM-1 (coactivator-associated arginine methyltransferase-1), DRIP-205 (vitaminD-receptor-interacting protein-205), SRC-1 (steroid receptor coactivator-1), and PRMT-1 (protein arginine methyl transferase-1) (66). It is unclear how these coactivators are recruited by FXR ligands and how they are involved in specific gene regulation by FXR ligands.

The relevance of FXR to hepatic physiology and disease is known from the observation that FXR-deleted mice on a high-fat diet exhibit hyperlipidemia and massive hepatic steatosis, as well as necroinflammation and fibrogenesis $(67,68)$. Importantly, when mice knocked-out for LDL receptor (LDLR) and FXR were challenged with fat, high fat led to macrosteatosis without inflammation in the livers of $\mathrm{LDLR}^{-/-} / \mathrm{FXR}^{+/+}$mice, whereas the livers of double-knockout ( $\mathrm{LDLR}^{-/-} / \mathrm{FXR}^{-/-}$) mice showed necroinflammation and increased hepatic levels of tumor necrosis factor- $\alpha$, intercellular adhesion molecule-1, transforming growth factor (TGF) $-\beta$, procollagen $1 \alpha 1$ and collagen, suggesting FXR may prevent progression of simple steatosis to NASH.

The molecular basis for these actions is being unraveled. FXR induces expression of genes that promote triglyceride clearance and mitochondrial fatty acid $\beta$-oxidation, together with a suppression of lipogenic gene transcription (69). Furthermore, small heterodimer partner (SHP), a nuclear receptor with corepressor activity that is strongly induced by FXR, interferes with SREBP-1c expression by inhibiting the activity of the liver $\mathrm{X}$ receptor, further reducing hepatic lipogenesis (70). FXR also upregulates the genes encoding apolipoprotein C-II (apoC-II) and very-low-density lipoprotein receptor, therefore enhancing triglyceride-rich lipoprotein clearance, while apoA-I gene expression is repressed (71). The latter action may explain the high-density-lipoprotein (HDL) cholesterol reduction observed in patients on bile acid supplementation, and the specular increase in apoA-I and HDL cholesterol levels in subjects after ileal resection or on cholestyramine (72).

FXR regulates glucose homeostasis as well. In mice, FXR deletion promotes insulin resistance in both muscle and liver, leading to impaired glucose tolerance and hyperglycemia. Synthetic FXR agonists decrease gluconeogenesis in mice fed a high-fat diet by reducing the expression and activity of PEPCK, G6Pase, and fructose-1,6-biphosphatase, and by enhancing insulin signaling through insulinreceptor substrate-1 (IRS-1) phosphorylation $(73,74)$.

In addition to its effects on glucose and lipid homeostasis, FXR appears to antagonize hepatic inflammatory and fibrogenetic processes. FXR agonists have been recently shown to antagonize nuclear factor (NF) $-\mathrm{KB}$ pathway activation in primary hepatocytes and in rodents challenged with bacterial lipopolysaccharide (LPS) (75). $\mathrm{FXR}^{-/-}$livers showed massive necroinflammation after treatment with LPS at a dose that did not induce significant liver damage in wild-type mice, whereas FXR activation suppressed NF- $\mathrm{kB}$ mediated expression of inducible nitric oxide synthase (iNOS), cyclooxygenase-2 (COX-2), interferon- $\gamma$-inducible protein-10 (IP-10), and interferon- $\gamma($ IFN- $\gamma)$ induced by LPS administration. Another hepatic protective mechanism of FXR activation against gut-derived endotoxins is represented by the maintenance of gut integrity through induction of antibacterial factors such as angiogenin, iNOS, and interleukin (IL)-18 (76). FXR also possesses antifibrotic properties. Synthetic FXR agonist 6-ethyl chenodeoxycholic acid (6-ECDCA) reduced the expression of the profibrotic genes encoding collagen $\alpha(1) \mathrm{I}, \alpha$-smooth muscle 
actin, tissue inhibitors of metalloproteinase 1 and 2 , and TGF- $\beta 1$ in cultured hepatic stellate cells. 6-ECDCA also reversed hepatic fibrosis in several rat models of liver injury $(77,78)$.

\section{NUCLEAR TRANSCRIPTION FACTORS: PREGNANE $X$ RECEPTOR}

The pregnane $\mathrm{X}$ receptor (PXR) was initially identified as a nuclear receptor regulating xenobiotic and drug metabolism and elimination (79). Upon activation by a variety of ligands including drugs, insecticides, pesticides, and nutritional compounds, PXR forms a heterodimer with RXR that binds to PXR response elements, located in the $5^{\prime}$-flanking regions of target genes, resulting in their transcriptional activation. This "xenosensor" coordinates the expression of several genes encoding the most important xenobiotic metabolizing and transporting system, to inactivate and eliminate these compounds through four phases: uptake; oxidation (mostly through cytochrome $\mathrm{P} 4503 \mathrm{~A}$ and 2B); conjugation by uridine-50-diphosphate glucuronosyltransferases (UGT), glutathione S-transferases (GST), or sulfono-transferases; and efflux, performed by transporters like multidrug resistance protein-1 (MDR1) (80). It subsequently became clear that this system was also involved in catabolism of bile acid, bilirubin, and thyroid and steroid hormone, affecting their kinetics (81) (Tables 4 and 5).

Subsequent functional studies using PXR knockout and transgenic mice have implicated PXR in hepatic steatosis and carbohydrate and lipid homeostasis. Transgenic mice overexpressing the activated human PXR in the liver displayed marked hepatic triglyceride accumulation compared to the wild type (82). The lipogenic effect of PXR was independent of SREBP-1c and its primary target enzymes FAS and ACC-1. This effect occurred at 3 levels (83):

1. Enhanced de novo lipogenesis. PXR activation directly enhanced transcription of lipogenic enzymes stearoylCoA desaturase-1 (SCD-1) and fatty acid elongase (FAE). Furthermore, PXR enhanced transcription of lipogenic enzymes FAS and ATP citrate lyase (ACLY) by interacting with the thyroid-hormoneresponsive SPOT14 homolog S14, a transcription regulator that is emerging as a key stimulator of lipogenesis induced by carbohydrate feeding and thyroid hormone administration (84).

2. Enhanced hepatocyte uptake of circulating FFA by the membrane fatty acid translocase fatty acid transporter (FAT)/CD36. Analysis of the mouse CD36 gene promoter revealed that CD36 is a direct transcriptional target of PXR. This regulation is achieved by binding of the PXR-RXR heterodimers to a PXR response element found in the CD36 gene promoter. Interestingly, the regulation of CD36 by PXR is liver specific. The same study found that activation of PXR also induced the expression of $\operatorname{PPAR} \gamma$, another positive regulator of CD36, suggesting that PXR can upregulate CD36 directly or indirectly through activation of PPAR $\gamma$ (82).

3. Downregulation of several genes involved in $\beta$-oxidation, including PPAR $\alpha$ and thiolase (82-85). The notion that activation of PXR may suppress $\beta$-oxidation and influence lipogenesis was supported by a recent study by Nakamura et al. (86). These authors showed that treatment with PXR agonist pregnenolone-16 $\alpha$ carbonitrile (PCN) downregulated transcription of the genes encoding carnitine palmitoyltransferase $1 \mathrm{a}$ (CPT1a) and mitochondrial 3-hydroxy3-methylglutarate-CoA synthase 2 (Hmgcs2) in the liver of fasting wildtype, but not PXR-null, mice (87). CPT1a is a key regulatory factor in insulin-dependent repression of $\beta$ oxidation, and Hmges2 is an enzyme important for ketogenesis. The SCD-1 mRNA was also found to be upregulated in PCN-treated wild-type mice, contributing to hepatic fatty infiltration. Consistent with the pattern of gene 
Table 4 Molecular mechanisms of action of farnesoid $\mathrm{X}$ receptor

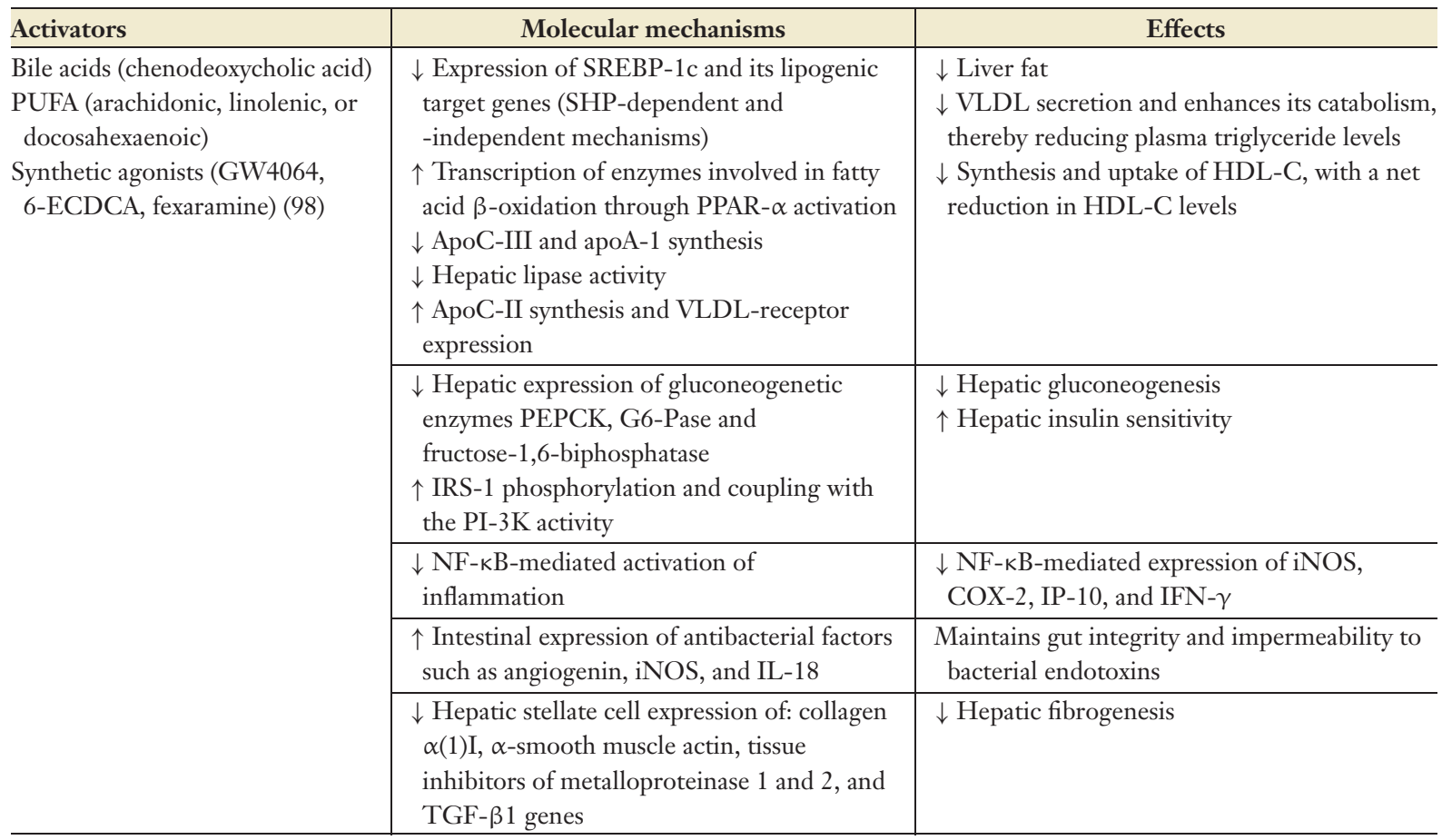

Abbreviations: PUFA, polyunsaturated fatty acid; 6-ECDCA, 6-ethyl chenodeoxycholic acid; SREBP-1c, sterol-response-element binding protein 1c; SHP, small heterodimer partner; VLDL, very-low-density lipoprotein; HDL, high-density lipoprotein; PPAR, peroxisome-proliferator-activated receptor; iNOS, inducible nitric oxide synthase; NF- $\mathrm{B}$, nuclear factor $\mathrm{\kappa B}$; TGF- $\beta 1$, transforming growth factor $\beta 1$; COX-2, cyclooxygenase-2; IP-10, interferon- $\gamma$-inducible protein 10; PEPCK, phosphoenol-pyruvate carboxykinase; IRS-1, insulin-receptor substrate-1; PI-3K, phosphoinositide 3-kinase.

Table 5 Molecular mechanisms of action of pregnane $\mathrm{X}$ receptor

\begin{tabular}{|c|c|c|}
\hline Activators & Molecular mechanisms & Effects \\
\hline $\begin{array}{l}5 \alpha \text {-pregnane-3,20-dione, } \\
\text { progesterone, } \\
17 \alpha \text {-hydoxyprogesterone, } \\
17 \alpha \text {-hydroxypregnenolone and } \\
\text { corticosterone, dexamethasone } \\
\text { Antiglucocorticoids (pregnenolone- } \\
16 \alpha \text {-carbonitrile, RU486) } \\
\text { Tamoxifen }\end{array}$ & $\begin{array}{l}\uparrow \text { Transcription of lipogenic enzymes SCD-1 and fatty } \\
\text { acid elongase } \\
\uparrow \text { Transcription of lipogenic enzymes FAS and ATP } \\
\text { citrate lyase (S14-mediated) } \\
\uparrow \text { Hepatocyte FFA uptake from plasma by FAT/CD36 } \\
\downarrow \text { Carnitine palmitoyltransferase 1A (CPT1a), } \\
\downarrow \text { PPAR- } \alpha \text { activation } \\
\downarrow \text { Glucogenesis }\end{array}$ & $\begin{array}{l}\uparrow \text { De novo fatty acid synthesis and } \\
\text { uptake from plasma } \\
\downarrow \text { Mitochondrial fatty acid } \\
\text { oxidation }\end{array}$ \\
\hline $\begin{array}{l}\text { Taxol } \\
\text { Anti-infective chemotherapies } \\
\text { (rifampicin, ritonavir, clotrimazole) }\end{array}$ & $\begin{array}{l}\downarrow \text { Hepatic expression of PEPCK and G6-Pase } \\
\text { (HNF-4/PGC- } 1 \alpha \text {-mediated) } \\
\downarrow \text { Hepatic FOXO1 transcription }\end{array}$ & $\downarrow$ Hepatic gluconeogenesis \\
\hline Organochlorine pesticides & $\begin{array}{l}\downarrow \text { Hepatic stellate cell transdifferentiation, } \\
\text { proliferation and fibrogenetic activity }\end{array}$ & $\downarrow$ Hepatic fibrogenesis \\
\hline
\end{tabular}

Abbreviations: SCD-1, stearoyl-CoA desaturase-1; FFA, free fatty acid; FAS, fatty acid synthase; FAT, fatty acid transporter; HNF-4, hepatocyte nuclear factor-4; PGC-1 $\alpha$, PPAR $\gamma$ coactivator $1 \alpha$; FOXO1, forkhead box-containing protein O subfamily-1. 
regulation, the levels of hepatic triglycerides were increased and levels of serum 3-hydroxybutyrate were decreased in the PCN-treated wild-type mice. The same study showed that PXR could cross-talk with the insulin-responsive forkhead factor FoxA2 to suppress the transcription of the CPT1a and Hmgcs2 genes. The authors concluded that activation of PXR represses FoxA2-mediated transcription of CPT1a and Hmges2 genes in fasting liver.

PXR suppresses hepatic gluconeogenesis by competing with hepatocyte nuclear factor-4 (HNF-4) for the binding of PPAR $\gamma$ coactivator$1 \alpha$ (PGC- $1 \alpha)$, thus attenuating HNF-4 signaling (88). The HNF-4/PGC-1 $\alpha$ pathway plays a central role in regulating glucose/energy metabolism by regulating the gluconeogenic G6-Pase and PEPCK genes (89). PXR also acts as a corepressor of the transcription factor forkhead box-containing protein $\mathrm{O}$ subfamily-1 (FOXO1), another stimulator of gluconeogenesis. FOXO1 overexpression in NASH correlated with the severity of hepatic necoinflammation and insulin resistance (90, 91).

Besides regulating lipid metabolism, PXR has been proposed as a potential target for antifibrotic therapy. In a rat model of carbon tetrachloride-induced injury, hepatocyte necrosis and liver fibrosis were significantly inhibited by PXR agonists (92). These effects were replicated in cultured human primary hepatic stellate cells, where the PXR ligand rifampicin inhibited the expression of fibrogenetic genes and reduced the proliferation and transdifferentiation to a profibrogenic phenotype of hepatic stellate cells. All of these effects were PXR dependent (93). The balance between the steatogenic and the antigluconeogenetic and antifibrotic effects of PXR activation must be weighed in a clinical model before any conclusion can drawn on the efficacy/safety profile of PXR antagonists as antifibrotic therapy.

\section{SUMMARY AND FUTURE DIRECTIONS}

With the growing epidemic of obesity, the prevalence of NAFLD will grow exponentially in the next 10 years. In the absence of an effective therapy, NASH is projected to be the leading cause of cirrhosis and liver transplantation by 2020 . Ongoing research faces every aspect of the putative pathogenetic mechanisms of NAFLD, from insulin resistance to dysregulated hepatic lipid and glucose metabolism to increased hepatic inflammation and fibrogenesis. Ongoing research targets molecular mechanisms at the crossroads of multiple abnormalities accompanying NAFLD: hepatic glucose and triglyceride dysmetabolism, liver fat infiltration, inflammation, and fibrosis. As of May 2009 , results of preclinical studies are encouraging. Two main challenges remain for the future: translating cellular/animal models to human pathology and testing the long-term safety and efficacy of those treatments that prove effective in preclinical trials.

\section{DISCLOSURE STATEMENT}

The authors are not aware of any affiliations, memberships, funding, or financial holdings that might be perceived as affecting the objectivity of this review.

\section{ACKNOWLEDGMENTS}

This work was supported by grants from Piedmont Regional Research Project Funds CIPE 2008. 


\section{LITERATURE CITED}

1. Szczepaniak LS, Nurenberg P, Leonard D, et al. 2005. Magnetic resonance spectroscopy to measure hepatic triglyceride content: prevalence of hepatic steatosis in the general population. Am. F. Physiol. Endocrinol. Metab. 288:E462-68

2. Ekstedt M, Franzén LE, Mathiesen UL, et al. 2006. Long-term follow-up of patients with NAFLD and elevated liver enzymes. Hepatology 44:865-73

3. Lee RC, Feinbaum RL, Ambros V. 1993. The C. elegans heterochronic gene lin-4 encodes small RNAs with antisense complementarity to lin-14. Cell 75:843-54

4. Griffiths-Jones S, Saini HK, van Dongen S, et al. 2008. miRBase: tools for microRNA genomics. Nucleic Acids Res. 36:D154-58

5. Selbach M, Schwanhäusser B, Thierfelder N, et al. 2008. Widespread changes in protein synthesis induced by microRNAs. Nature 455:58-63

6. Ding XC, Weiler J, Großhans H. 2008. Regulating the regulators: mechanisms controlling the maturation of microRNAs. Trends Biotechnol. 27:27-36

7. Zhang C. 2008. MicroRNAs: role in cardiovascular biology and disease. Clin. Sci. 114:699-706

8. Ladeiro Y, Couchy G, Balabaud C, et al. 2008. MicroRNA profiling in hepatocellular tumors is associated to clinical features and oncogene/tumor suppressor gene mutations. Hepatology 47:1955-63

9. Mott JL. 2009. MicroRNAs involved in tumor suppressor and oncogene pathways: implications for hepatobiliary neoplasia. Hepatology 50(2):630-67

10. Varnholt H. 2008. The role of microRNAs in primary liver cancer. Ann. Hepatol. 7:104-113

11. Jopling CL, Yi M, Lancaster AM, et al. 2005. Modulation of hepatitis C virus RNA abundance by a liver-specific MicroRNA. Science 309:1577-81

12. Ura S, Honda M, Yamashita T, et al. 2009. Differential microRNA expression between hepatitis B and hepatitis C leading disease progression to hepatocellular carcinoma. Hepatology 49:1098-112

13. Pedersen IM, Cheng G, Wieland S, et al. 2007. Interferon modulation of cellular microRNAs as an antiviral mechanism. Nature 449:919-22

14. Vinciguerra M, Sgroi A, Veyrat-Durebex C, et al. 2009. Unsaturated fatty acids inhibit the expression of tumor suppressor phosphatase and tensin homolog (PTEN) via microRNA-21 up-regulation in hepatocytes. Hepatology 49:1176-84

15. Horie Y, Suzuki A, Kataoka E, et al. 2004. Hepatocyte-specific Pten deficiency results in steatohepatitis and hepatocellular carcinomas. F. Clin. Invest. 113:1774-83

16. Jin X, Ye YF, Chen SH, et al. 2009. MicroRNA expression pattern in different stages of nonalcoholic fatty liver disease. Dig. Liver Dis. 41:289-97

17. Cheung O, Puri P, Eicken C, et al. 2008. Nonalcoholic steatohepatitis is associated with altered hepatic microRNA expression. Hepatology 48:1810-20

18. Elmén J, Lindow M, Schütz S, et al. 2008. LNA-mediated microRNA silencing in nonhuman primates. Nature 452:896-99

19. Krutzfeldt J, Rajewsky N, Braich R, et al. 2005. Silencing of microRNAs in vivo with 'antagomirs'. Nature 438:685-89

20. Esau C, Davis S, Murray SF, et al. 2006. miR-122 regulation of lipid metabolism revealed by in vivo antisense targeting. Cell Metab. 3:87-98

21. Esquela-Kerscher A, Trang P, Wiggins JF, et al. 2008. The let-7 microRNA reduces tumor growth in mouse models of lung cancer. Cell Cycle 7:759-64

22. Baggio LR, Drucker DJ. 2006. Therapeutic approaches to preserve islet mass in type 2 diabetes. Annu. Rev. Med. 57:265-81

23. Musso G, Gambino R, Pacini G, et al. 2009. Transcription factor 7-like 2 polymorphism modulates glucose and lipid homeostasis, adipokine profile, and hepatocyte apoptosis in NASH. Hepatology 49:426-35

24. Ding X, Saxena NK, Lin S, et al. 2006. Exendin-4, a glucagon-like protein-1 (GLP-1) receptor agonist, reverses hepatic steatosis in ob/ob mice. Hepatology 43:173-81

25. Tushuizen ME, Bunck MC, Pouwels PJ, et al. 2006. Incretin mimetics as a novel therapeutic option for hepatic steatosis. Liver Int. 26:1015-17 
26. Klonoff DC, Buse JB, Nielsen LL, et al. 2008. Exenatide effects on diabetes, obesity, cardiovascular risk factors and hepatic biomarkers in patients with type 2 diabetes treated for at least 3 years. Curr. Med. Res. Opin. 24:275-86

27. Flatt PR. 2007. Effective surgical treatment of obesity may be mediated by ablation of the lipogenic gut hormone gastric inhibitory polipeptide (GIP): evidence and clinical opportunity for development of new obesity-diabetes drugs? Diabetes Vasc. Dis. Res. 4:150-52

28. Baggio LL, Drucker DJ. 2007. Biology of incretins: GLP-1 and GIP. Gastroenterology 132:2131-57

29. Kim SJ, Nian C, McIntosh CH. 2007. Resistin is a key mediator of glucose-dependent insulinotropic polypeptide (GIP) stimulation of lipoprotein lipase (LPL) activity in adipocytes. F. Biol. Chem. 282:3413947

30. Hansotia T, Maida A, Flock G, et al. 2007. Extrapancreatic incretin receptors modulate glucose homeostasis, body weight, and energy expenditure. 7. Clin. Invest. 117:143-52

31. Gault VA, McClean PL, Cassidy RS, et al. 2007. Chemical gastric inhibitory polypeptide receptor antagonism protects against obesity, insulin resistance, glucose intolerance and associated disturbances in mice fed high-fat and cafeteria diets. Diabetologia 50:1752-62

32. McClean P, Irwin N, Cassidy RS, et al. 2007. GIP receptor antagonism reverses obesity, insulin resistance and associated metabolic disturbances induced in mice by prolonged consumption of high fat diet. Am. F. Physiol. Endocrinol. Metab. 293:E1746-55

33. Yamada Y, Miyawaki K, Tsukiyama K, et al. 2006. Pancreatic and extrapancreatic effects of gastric inhibitory polypeptide. Diabetes 55(Suppl. 2):S86-91

34. Thomsen C, Rasmussen O, Lousen T, et al. 1999. Differential effects of saturated and monounsaturated fatty acids on postprandial lipemia and incretin responses in healthy subjects. Am. 7. Clin. Nutr. 69:1135-43

35. Arsov T, Carter CZ, Nolan CJ, et al. 2006. Adaptive failure to high-fat diet characterizes steatohepatitis in Alms1 mutant mice. Biochem. Biophys. Res. Comm. 342:1152-59

36. Robertson RP, Harmon J, Tran POT, et al. 2004. $\beta$-Cell glucose toxicity, lipotoxicity, and chronic oxidative stress in type 2 diabetes. Diabetes 53:S119-24

37. Harding AH, Day NE, Khaw KT, et al. 2004. Dietary fat and the risk of clinical type 2 diabetes: the European Prospective Investigation of Cancer-Norfolk Study. Am. 7. Epidemiol. 159:73-82

38. Song DH, Getty-Haushik L, Tseng E, et al. 2007. Glucose-dependent insulinotropic polypeptide enhances adipocyte development and glucose uptake in part through Akt activation. Gastroenterology 133:1796-805

39. Musso G, Gambino R, Pacini G, et al. 2009. Prolonged saturated fat-induced, glucose-dependent insulinotropic polypeptide elevation is associated with adipokine imbalance and liver injury in nonalcoholic steatohepatitis: dysregulated enteroadipocyte axis as a novel feature of fatty liver. Am. F. Clin. Nutr. 89:55867

40. Lamont BJ, Drucker DJ. 2008. Differential antidiabetic efficacy of incretin agonists versus DPP-4 inhibition in high fat fed mice. Diabetes 57:190-98

41. Suckling K. 2008. Selective thyromimetics for atherosclerosis and dyslipidaemia: another old target making progress. Expert Opin. Invest. Drugs 17:615-18

42. Ribeiro MO. 2008. Effects of thyroid hormone analogs on lipid metabolism and thermogenesis. Thyroid 18:197-203

43. Berkenstam A, Kristensen J, Mellstrom K, et al. 2008. The thyroid hormone mimetic compound KB2115 lowers plasma LDL cholesterol and stimulates bile acid synthesis without cardiac effects in humans. Proc. Natl. Acad. Sci. USA 105:663-67

44. Cable EE, Finn PD, Stebbins JW, et al. 2009. Reduction of hepatic steatosis in rats and mice after treatment with a liver-targeted thyroid hormone receptor agonist. Hepatology 49:407-17

45. Perra A, Simbula G, Simbula M, et al. 2008. Thyroid hormone (T3) and TRbeta agonist GC-1 inhibit/reverse nonalcoholic fatty liver in rats. FASEB 7. 22:2981-89

46. Grant N. 2007. The role of triiodothyronine-induced substrate cycles in the hepatic response to overnutrition: thyroid hormone as an antioxidant. Med. Hypotheses 68:641-49

47. Viollet B, Lantier L, Devin-Leclerc J, et al. 2009. Targeting the AMPK pathway for the treatment of type 2 diabetes. Front. Biosci. 14:3380-400

48. Suter M, Riek U, Tuerk R, et al. 2006. Dissecting the role of 5'-AMP for allosteric stimulation, activation, and deactivation of AMP-activated protein kinase. F. Biol. Chem. 281(43):32207-16 
49. Iglesias MA, Ye JM, Frangioudakis G, et al. 2002. AICAR administration causes an apparent enhancement of muscle and liver insulin action in insulin-resistant high-fat-fed rats. Diabetes 51:2886-94

50. Koo SH, Flechner L, Qi L, et al. 2005. The CREB coactivator TORC2 is a key regulator of fasting glucose metabolism. Nature 437:1109-11

51. Taylor EB, An D, Kramer HF, et al. 2008. Discovery of TBC1D1 as an insulin-, AICAR-, and contractionstimulated signaling nexus in mouse skeletal muscle. F. Biol. Chem. 283:9787-96

52. Bergeron R, Previs SF, Cline GW, et al. 2001. Effect of 5-aminoimidazole-4-carboxamide-1-beta-Dribofuranoside infusion on in vivo glucose and lipid metabolism in lean and obese Zucker rats. Diabetes 50:1076-82

53. Cool B, Zinker B, Chiou W, et al. 2006. Identification and characterization of a small molecule AMPK activator that treats key components of type 2 diabetes and the metabolic syndrome. Cell Metab. 3:403-16

54. Park KG, Min AK, Koh EH, et al. 2008. Alpha-lipoic acid decreases hepatic lipogenesis through adenosine monophosphate-activated protein kinase (AMPK)-dependent and AMPK-independent pathways. Hepatology 48:1477-86

55. Hwahng SH, Ki SH, Bae EJ, et al. 2009. Role of adenosine monophosphate-activated protein kinase-p70 ribosomal S6 kinase-1 pathway in repression of liver X receptor-alpha-dependent lipogenic gene induction and hepatic steatosis by a novel class of dithiolethiones. Hepatology 49:1913-25

56. Lagouge M, Argmann C, Gerhart-Hines Z, et al. 2006. Resveratrol improves mitochondrial function and protects against metabolic disease by activating SIRT1 and PGC-1alpha. Cell 127:1109-22

57. Minokoshi Y, Alquier T, Furukawa N, et al. 2004. AMP-kinase regulates food intake by responding to hormonal and nutrient signals in the hypothalamus. Nature 428:569-74

58. Nishino Y, Miura T, Miki T, et al. 2004. Ischemic preconditioning activates AMPK in a PKC-dependent manner and induces GLUT4 upregulation in the late phase of cardioprotection. Cardiovasc. Res. 61:610-19

59. Shibata R, Ouchi N, Ito M, et al. 2004. Adiponectin-mediated modulation of hypertrophic signals in the heart. Nat. Med. 10:1384-89

60. Majithiya JB, Balaraman R. 2006. Metformin reduces blood pressure and restores endothelial function in aorta of streptozotocin-induced diabetic rats. Life Sci. 78:2615-24

61. Pold R, Jensen LS, Jessen N, et al. 2005. Long-term AICAR administration and exercise prevents diabetes in ZDF rats. Diabetes 54:928-34

62. Boon H, Bosselaar M, Praet SF, et al. 2008. Intravenous AICAR administration reduces hepatic glucose output and inhibits whole body lipolysis in type 2 diabetic patients. Diabetologia 51:1893-900

63. Parks DJ, Blanchard SG, Bledsoe RK, et al. 1999. Bile acids: natural ligands for an orphan nuclear receptor. Science 284:1365-68

64. Downes M, Verdecia MA, Roecker AJ, et al. 2003. A chemical, genetic, and structural analysis of the nuclear bile acid receptor FXR. Mol. Cell 11:1079-92

65. Thomas C, Pellicciari R, Pruzanski M, et al. 2008. Targeting bile-acid signaling for metabolic diseases. Nat. Rev. 7:678-93

66. Savkur RS, Thomas JS, Bramlett KS, et al. 2005. Ligand-dependent coactivation of the human bile acid receptor FXR by the peroxisome proliferator-activated receptor gamma coactivator-1alpha. F. Pharmacol. Exp. Ther. 312:170-78

67. Sinal CJ, Tohkin M, Miyata M, et al. 2000. Targeted disruption of the nuclear receptor FXR/BAR impairs bile acid and lipid homeostasis. Cell 102:731-44

68. Kong B, Luyendyk JP, Tawfik O, et al. 2009. Farnesoid X receptor deficiency induces nonalcoholic steatohepatitis in low-density lipoprotein receptor-knockout mice fed a high-fat diet. F. Pharmacol. Exp. Ther: 328:116-22

69. Zhang Y, Castellani LW, Sinal CJ, et al. 2004. Peroxisome proliferator-activated receptor-gamma coactivator $1 \alpha(\mathrm{PGC}-1 \alpha)$ regulates triglyceride metabolism by activation of the nuclear receptor FXR. Genes Dev. 18:157-69

70. Watanabe M, Houten SM, Wang L, et al. 2004. Bile acids lower triglyceride levels via a pathway involving FXR, SHP, and SREBP-1c. F. Clin. Invest. 113:1408-18

71. Claudel T, Inoue Y, Barbier O, et al. 2003. Farnesoid X receptor agonists suppress hepatic apolipoprotein CIII expression. Gastroenterology 125:544-55 
72. Buchwald H, Varco RL, Matts JP, et al. 1990. Effect of partial ileal bypass surgery on mortality and morbidity from coronary heart disease in patients with hypercholesterolemia. Report of the Program on the Surgical Control of the Hyperlipidemias (POSCH). N. Engl. F. Med. 323:946-55

73. Yamagata K, Daitoku H, Shimamoto Y, et al. 2004. Bile acids regulate gluconeogenic gene expression via small heterodimer partner-mediated repression of hepatocyte nuclear factor 4 and Foxo1. 7. Biol. Chem. 279:23158-65

74. Ma K, Saha PK, Chan L, et al. 2006. Farnesoid X receptor is essential for normal glucose homeostasis. 7. Clin. Invest. 116:1102-9

75. Wang YD, Chen WD, Wang M, et al. 2008. Farnesoid X receptor antagonizes NF- $\mathrm{B}$ in hepatic inflammatory response. Hepatology 48:1632-43

76. Inagaki T, Moschetta A, Lee YK, et al. 2006. Regulation of antibacterial defense in the small intestine by the nuclear bile acid receptor. Proc. Natl. Acad. Sci. USA 103:3920-25

77. Fiorucci S, Antonelli E, Rizzo G, et al. 2004. The nuclear receptor SHP mediates inhibition of hepatic stellate cells by FXR and protects against liver fibrosis. Gastroenterology 127:1497-512

78. Fiorucci S, Rizzo G, Antonelli E, et al. 2005. A farnesoid X receptor-small heterodimer partner regulatory cascade modulates tissue metalloproteinase inhibitor-1 and matrix metalloprotease expression in hepatic stellate cells and promotes resolution of liver fibrosis. F. Pharmacol. Exp. Ther: 314:584-95

79. Blumberg B, Sabbagh W, Juguilon H, et al. 1998. SXR, a novel steroid and xenobiotic-sensing nuclear receptor. Genes Dev. 12:3195-205

80. Timsit YE, Negishi M. 2007. CAR and PXR: the xenobiotic-sensing receptors. Steroids 72:231-46

81. Pascussi JM, Gerbal-Chaloin S, Duret C, et al. 2008. The tangle of nuclear receptors that controls xenobiotic metabolism and transport: crosstalk and consequences. Annu. Rev. Pharmacol. Toxicol. 48:1-32

82. Zhou J, Febbraio M, Wada T, et al. 2008. Hepatic fatty acid transporter Cd36 is a common target of LXR, PXR, and PPAR $\gamma$ in promoting steatosis. Gastroenterology 134:556-67

83. Hariparsad N, Chu X, Yabut J, et al. 2009. Identification of pregnane-X receptor target genes and coactivator and corepressor binding to promoter elements in human hepatocytes. Nucleic Acids Res. 37:1160-73

84. Moreau A, Téruel C, Beylot M, et al. 2009. A novel pregnane X receptor and S14-mediated lipogenic pathway in human hepatocyte. Hepatology 49:2068-79

85. Hoekstra M, Lammers B, Out R, et al. 2009. Activation of the nuclear receptor PXR decreases plasma LDL-cholesterol levels and induces hepatic steatosis in LDL receptor knockout mice. Mol. Pharmacol. 6:182-89

86. Nakamura K, Moore R, Negishi M, et al. 2007. Nuclear pregnane X receptor cross-talk with FoxA2 to mediate drug-induced regulation of lipid metabolism in fasting mouse liver. F. Biol. Chem. 282:9768-76

87. Wolfrum C, Asilmaz E, Luca E, et al. 2004. Foxa2 regulates lipid metabolism and ketogenesis in the liver during fasting and in diabetes. Nature 432:1027-32

88. Bhalla S, Ozalp C, Fang S, et al. 2004. Ligand-activated pregnane X receptor interferes with HNF-4 signaling by targeting a common coactivator PGC-1alpha. Functional implications in hepatic cholesterol and glucose metabolism. 7. Biol. Chem. 279:45139-47

89. Miao J, Fang S, Bae Y, Kemper JK. 2006. Functional inhibitory cross-talk between constitutive androstane receptor and hepatic nuclear factor-4 in hepatic lipid/glucose metabolism is mediated by competition for binding to the DR1 motif and to the common coactivators, GRIP-1 and PGC-1alpha. 7. Biol. Chem. 281:14537-46

90. Valenti L, Rametta R, Dongiovanni P, et al. 2008. Increased expression and activity of the transcription factor FOXO1 in nonalcoholic steatohepatitis. Diabetes 57:1355-62

91. Kodama S, Koike C, Negishi M, et al. 2004. Nuclear receptors CAR and PXR cross talk with FOXO1 to regulate genes that encode drug-metabolizing and gluconeogenic enzymes. Mol. Cell. Biol. 24:7931-40

92. Marek CJ, Tucker SJ, Konstantinou DK, et al. 2005. Pregnenolone-16alpha-carbonitrile inhibits rodent liver fibrogenesis via PXR (pregnane X receptor)-dependent and PXR-independent mechanisms. Biochem. 7. 387(Pt. 3):601-8

93. Haughton EL, Tucker SJ, Marek CJ, et al. 2006. Pregnane X receptor activators inhibit human hepatic stellate cell transdifferentiation in vitro. Gastroenterology 131:194-209

94. Li L, Yang G, Li Q, et al. 2008. Exenatide prevents fat-induced insulin resistance and raises adiponectin expression and plasma levels. Diabetes Obes. Metab. 10:921-30 
95. Zang M, Xu S, Maitland-Toolan KA, et al. 2006. Polyphenols stimulate AMP-activated protein kinase, lower lipids, and inhibit accelerated atherosclerosis in diabetic LDL receptor-deficient mice. Diabetes 55:2180-91

96. Collins QF, Liu HY, Pi J, et al. 2007. Epigallocatechin-3-gallate (EGCG), a green tea polyphenol, suppresses hepatic gluconeogenesis through 5'-AMP-activated protein kinase. F. Biol. Chem. 282:30143-49

97. Jager S, Handschin C, St-Pierre J, et al. 2007. AMP-activated protein kinase (AMPK) action in skeletal muscle via direct phosphorylation of PGC-1alpha. Proc. Natl. Acad. Sci. USA 104:12017-22

98. Zhao A, Yu J, Lew JL, et al. 2004. Polyunsaturated fatty acids are FXR ligands and differentially regulate expression of FXR targets. DNA Cell Biol. 23:519-26 
Annual Review of

Medicine

\section{Contents}

Volume 61, 2010

Using Genetic Diagnosis to Determine Individual

Therapeutic Utility

C. Thomas Caskey

Emotion Recollected in Tranquility: Lessons Learned from the COX-2 Saga

Tilo Grosser, Ying Yu, and Garret A. FitzGerald

Progressive Multifocal Leukoencephalopathy in Patients on

Immunomodulatory Therapies

Eugene O. Major

The Future of Antiplatelet Therapy in Cardiovascular Disease

Carlo Patrono and Bianca Rocca...

Pharmacogenetics of Warfarin

Farbad Kamali and Hilary Wynne....

Heparin-Induced Thrombocytopenia

Gowthami M. Arepally and Thomas L. Ortel ......................................77

Regulation of Phosphate Homeostasis by PTH, Vitamin D, and FGF23

Clemens Bergwitz and Harald Füppner

Alveolar Surfactant Homeostasis and the Pathogenesis of Pulmonary

Disease

Feffrey A. Whitsett, Susan E. Wert, and Timothy E. Weaver

Diagnosis and Treatment of Neuropsychiatric Disorders

Katherine H. Taber, Robin A. Hurley, and Stuart C. Yudofsky....

Toward an Antibody-Based HIV-1 Vaccine

fames A. Hoxie

HIV-1 Vaccine Development After STEP

Dan H. Barouch and Bette Korber

Growing Up with HIV: Children, Adolescents, and Young Adults with

Perinatally Acquired HIV Infection

Rohan Hazra, George K. Siberry, and Lynne M. Mofenson 
H5N1 Avian Influenza: Preventive and Therapeutic Strategies

Against a Pandemic

Suryaprakash Sambhara and Gregory A. Poland

Revascularization for Coronary Artery Disease: Stents Versus Bypass

Surgery

Spencer B. King III, Fobn Feffrey Marshall, and Pradyumna E. Tummala

Controversies in the Use of Drug-Eluting Stents for Acute Myocardial

Infarction: A Critical Appraisal of the Data

Rabul Sakbuja and Laura Mauri

Arrythmogenic Cardiomyopathy: Etiology, Diagnosis, and Treatment

Srijita Sen-Chowdbry, Robert D. Morgan, John C. Chambers, and William 7. McKenna

Contemporary Use of Ventricular Assist Devices

Cesare M. Terracciano, Leslie W. Miller, and Magdi H. Yacoub ....

Stress Cardiomyopathy

Yoshibiro 7. Akashi, Holger M. Nef, Helge Möllmann, and Takashi Ueyama

Stem Cells in the Treatment of Heart Disease

Stefan Fanssens

Biological Mechanisms Linking Obesity and Cancer Risk:

New Perspectives

Darren L. Roberts, Caroline Dive, and Andrew G. Renehan

Hepatocellular Carcinoma: Novel Molecular Approaches for

Diagnosis, Prognosis, and Therapy

Augusto Villanueva, Beatriz Minguez, Alejandro Forner, Maria Reig,

and Fosep $M$. Llovet

Molecular Diagnosis and Therapy of Kidney Cancer

W. Marston Linehan, Gennady Bratslavsky, Peter A. Pinto, Laura S. Schmidt, Len Neckers, Donald P. Bottaro, and Ramaprasad Srinivasan

Myelodysplastic Syndromes

Bart L. Scott and H. Foachim Deeg

Nanotechnology Applications in Surgical Oncology

Sunil Singhal, Shuming Nie, and May D. Wang ....

Emerging Molecular Targets for the Treatment of Nonalcoholic Fatty

Liver Disease

Giovanni Musso, Roberto Gambino, and Maurizio Cassader

Metabolic Surgery to Treat Type 2 Diabetes: Clinical Outcomes and Mechanisms of Action

Francesco Rubino, Philip R. Schauer, Lee M. Kaplan, and David E. Cummings 
Genetic Aspects of Pancreatitis

David C. Whitcomb

Anorexia Nervosa: Current Status and Future Directions

Evelyn Attia

Structural Variation in the Human Genome and its Role in Disease

Pawel Stankiewicz and fames R. Lupski

Surgical Innovations Arising from the Iraq and Afghanistan Wars

Geoffrey S.F. Ling, Peter Rhee, and Fames M. Ecklund

Medicare Part D: Ongoing Challenges for Doctors and Patients

Gretchen Facobson and Gerard Anderson

\section{Indexes}

Cumulative Index of Contributing Authors, Volumes 57-61 ................... 477

Cumulative Index of Chapter Titles, Volumes $57-61 \ldots \ldots \ldots \ldots \ldots \ldots \ldots \ldots \ldots . . . \ldots 481$

\section{Errata}

An online log of corrections to Annual Review of Medicine articles may be found at http://med.annualreviews.org/errata.shtml 